\title{
EEG IN NONCONVULSIVE STATUS EPILEPTICUS
}

Ictal and interictal EEG findings and the diagnostic utility of iv diazepam in 78 patients with nonconvulsive status epilepticus (NCSE) are reported from the Department of Neurology, University of Virginia Health Sciences Center, Charlottesville, VA. Ictal discharges were generalized in 59 episodes $(69 \%)$ of NCSE, diffuse with focal predominance in 15 (18\%), and focal in 11 (13\%). EEG characteristics were heterogeneous. Atypical spike and wave was the predominant pattern, and typical spike and wave of absence seizures was rare. Rhythmic delta with intermittent spikes was most prevalent in the group with diffuse and focal discharges. Response to iv diazepam, 1 or $2 \mathrm{mg}$ every 30-60 sec, occurred at a dose $<8 \mathrm{mg}$. Focal NCSE was less likely to respond than generalized NCSE. Persistence of interictal focal discharges after diazepam may differentiate generalized from focal onset NCSE. (Granner MA, Lee SI. Nonconvulsive status epilepticus: EEG analysis in a large series. Epilepsia Jan/Feb 1994; $\underline{35}: 42-47$ ). (Reprints: Dr SI Lee, Department of Neurology, Box 394, University of Virginia, Health Sciences Center, Charlottesville, VA 22908).

COMMENT. NCSE is characterized by slowness in behavior and mentation, confusion, or stupor, lasting $>1$ hour and accompanied by EEG epileptiform, continuous activity, either generalized, focal, or focal with secondary generalization.

In a study of 253 adults with SE admitted to the Medical College of Virginia, Richmond, VA, mortality rates of patients with prolonged ( $>60$ min) compared to nonprolonged (30-59 min) status were significantly different (32\% of $2.7 \%$ ). Duration of status was a strong prognostic factor, while the type of seizure was not a determinant of mortality. The mortality rate was $30 \%$ in 70 patients with partial SE, and $20 \%$ in 180 with generalized SE. Anoxia and increasing age were significantly correlated with higher mortality. (Towne AR et al. Determinants of

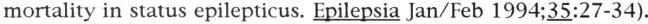

\section{SEIZURE DISORDERS}

\section{ICTUS EMETICUS INDUCED BY PHOTIC STIMULATION}

Occipitotemporal seizures induced by intermittent photic stimulation in three children with brain injuries, aged 10 to 13 years, are reported from the Institute of Developmental Neuropsychiatry, University of Pisa, Italy. Infantile or early childhood seizures (infantile spasms, febrile seizures, or complex partial seizures) had been controlled and medications discontinued. All had a history of occipital spikes on the EEG. Photic stimulation induced electroclinical phenomena localized to the right occipital lobe with spread to mesial temporal limbic structures, including amygdala and hippocampus. Symptoms began with blindness, tonic eye deviation, blinking, oral automatisms, epigastric sensations, fear, reduced responsiveness, rhythmic chewing, and vomiting, appearing as a late ictal manifestation. All patients were seizure free at 1 year follow up; 2 were untreated and one had received carbamazepine. Vomiting can be a late ictal phenomenon resulting from temporal lobe spread of seizures originating in the occipital lobe. (Guerrini $R$ et al. Occipitotemporal seizures with ictus emeticus induced by intermittent photic stimulation. Neurology Feb 1994;4ㅕ:253-259). (Reprints: Dr Renzo Guerrini, 